\title{
Practical solutions for reliable triple probe measurements in magnetized plasmas
}

\author{
C. Theiler, a) I. Furno, A. Kuenlin, Ph. Marmillod, and A. Fasoli \\ Centre de Recherches en Physique des Plasmas-Ecole Polytechnique Fédérale de Lausanne (EPFL), \\ Association EURATOM-Confédération Suisse, CH-1015 Lausanne, Switzerland
}

(Received 7 September 2010; accepted 24 October 2010; published online 13 January 2011)

\begin{abstract}
The triple probe method to obtain local, time-resolved measurements of density, electron temperature and plasma potential is investigated in detail. The difficulties in obtaining reliable measurements with this technique are discussed and overcome. These include phase delay errors, ion sheath expansion and limited bandwidth due to stray capacitance to ground. In particular, a relatively simple electronic circuit is described to strongly reduce stray capacitance. Measurements with the triple probe are presented in a plasma characterized by interchange-driven turbulence in the TORPEX device. The measured time-averaged and time-dependent, conditionally averaged parameters are cross-checked with other Langmuir probe based techniques, and show good agreement. Triple probe measurements show that electron temperature fluctuations are sufficiently large, such that the identification of plasma potential fluctuations with fluctuations of the floating potential is not a good approximation. Over a large radial region, the time-averaged fluctuation-induced particle flux can, however, be deduced from floating potential only. This is because the phase shift between density and electron temperature is close to zero there and temperature fluctuations do not give rise to a net radial particle transport. () 2011 American Institute of Physics. [doi:10.1063/1.3516045]
\end{abstract}

\section{INTRODUCTION}

The Langmuir probe (LP) is a widely used diagnostics to obtain local measurements of plasma density, electron temperature and plasma potential. It consists of a metal tip that is inserted into the plasma. A sweep of the potential applied to the tip allows obtaining the current-voltage $(I-V)$ characteristics. Depending on plasma parameters and probe geometry, different theories exist to interpret the $I-V$ characteristics and deduce plasma parameters. ${ }^{1-5}$ Usually, the probe is swept at a frequency much lower than that of typical plasma fluctuations and the $I-V$ curve is used to deduce time average quantities. Time-dependent measurements are often limited to ion-saturation current and floating potential measurements, i.e., to the current drawn from the plasma at a strong negative probe potential and the potential for which the probe draws no current, respectively.

Although rapidly swept LP's are being used, ${ }^{6,7}$ probably the most common method to obtain local, time-dependent measurements of plasma density, electron temperature and plasma potential is the triple probe technique. ${ }^{8}$ In the simplest configuration, it consists of three Langmuir probe tips, which instantaneously record three points of the $I-V$ characteristics. This allows a direct evaluation of the above quantities, provided that all probes see the same plasma conditions. To come close to this ideal situation, the probe tips are usually aligned perpendicular to background plasma gradients. In order to avoid shadowing effects, they are sufficiently spaced and, in the presence of a magnetic field, positioned on separate field lines. However, other effects such as phase delay errors, ion-sheath expansion and stray capacitance can affect the measurements. ${ }^{5,8,9}$

a) Electronic mail: christian.theiler@epfl.ch.
In this paper, we present a detailed study of the triple probe technique in a magnetized plasma in the TORPEX (Ref. 10) device whose background (time average) and fluctuation properties are well characterized. In Sec. II, we briefly discuss the basic principle of the triple probe. We then discuss methods to account for phase delay errors and ion-sheath expansion effects. We also point out how plasma parameters affect the bandwidth of the measurements. In Sec. III, we describe a novel guarding circuitry that reduces stray capacitance and improves the bandwidth. Design and construction of the probe used in this work are illustrated in Sec. IV. In Sec. V, we review the interchange-driven plasma configuration in the TORPEX device, in which the probe is tested. We start the discussion of the experimental results with consistency tests of the probe (Sec. V A) and cross-checks with other LP-based methods (Sec. V B) before presenting the main results obtained with the new triple probe (Sec. V C). The conclusions in Sec. VI are followed by a discussion of the single-LP theory used in TORPEX to interpret probe data (the Appendix).

\section{TRIPLE PROBE PRINCIPLE}

The standard triple probe consists of three probe tips, i.e., tips 1-3 in the scheme shown in Fig. 1, with switch A closed and switch B open. Probe 1 measures the floating potential $V_{\mathrm{fl}}$ while the other two tips form a double probe circuit. ${ }^{11} \mathrm{~A}$ constant potential difference $\Delta V$ is applied between the two tips and the potentials $V^{+}$and $V^{-}$adjust to satisfy current continuity in the double probe circuit. In an ideal situation and for sufficiently large values of $\Delta V\left(\Delta V \gg T_{\mathrm{e}} / e\right)$, the electron temperature $T_{\mathrm{e}}$, density $n$ and plasma potential $V_{\mathrm{pl}}$ are 


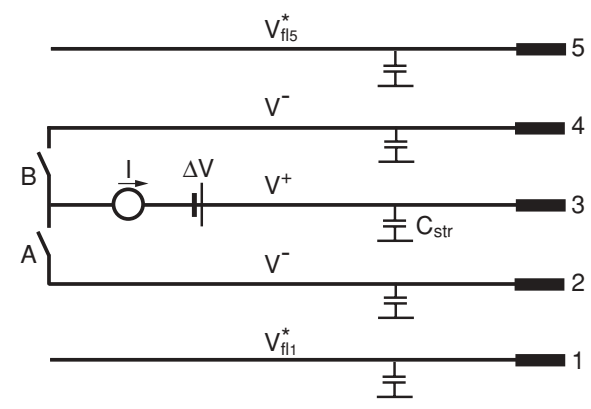

FIG. 1. Scheme of the triple probe method. Probe tips $1-5$ are indicated together with the quantities they measure. The switches A and B allow changing between the standard triple probe method and the modified fivetip method. The capacitances $C_{\text {str }}$ indicate undesired stray capacitances in the circuit.

given by

$$
T_{\mathrm{e}}=\frac{e\left(V^{+}-V_{\mathrm{fl}}\right)}{\ln (2)}, \quad n=\frac{2 I}{e c_{s} A}, \quad V_{\mathrm{pl}}=V_{\mathrm{fl}}+\mu T_{\mathrm{e}} / e .
$$

Here, $e$ is the proton charge, $c_{s} \approx \sqrt{T_{\mathrm{e}} / m_{i}}$ the ion sound speed for cold ions, $m_{i}$ the ion mass, $A$ the surface of the probe, $I$ the current flowing in the double probe circuit and $\mu$ a parameter that can be evaluated experimentally for a given probe $^{12}$ (typically $\approx 3$ for hydrogen plasmas ${ }^{4}$ ). Quantities are expressed in SI units. $T_{e}$ stands for $k_{B} T_{e}$.

However, even if the three probe tips are aligned perpendicularly to the background plasma gradients and they measure the same time-averaged parameters, the instantaneous parameters can vary from tip to tip. A wave propagating along the direction of the tip alignment for example leads to phase delay errors and erroneous measurements. This problem can be addressed by the modified triple probe method, ${ }^{9}$ which uses a symmetric arrangement with five probe tips. This corresponds to the configuration in Fig. 1 when both switches A $\mathrm{B}$ are closed. The two additional tips (4 and 5) provide conjugate phase information to reduce phase delay errors in the standard three tip configuration to second order in $k d$, with $k$ the perpendicular wave number and $d$ the tip spacing. ${ }^{9}$

Tips 1 and 5 measure the floating potential. These measurements are combined to evaluate the floating potential at the center (tip 3). The two signals are acquired independently. This allows us to evaluate gradients in the floating potential, to estimate the vertical wave number $k_{z}(f)$ as a function of frequency from two-point correlation ${ }^{13}$ and the amplitude reduction of floating potential fluctuations due to the averaging of the two signals and the error caused by this (see the particle transport analysis in Sec. V C).

The tips 2-4 form the double probe circuit. Besides $V^{+}$ and $V^{-}=V^{+}-\Delta V$, the current $I$ flowing through the circuit is also measured. Assuming that phase delay errors have been reduced sufficiently and that all tips are identical, the time evolution of $T_{\mathrm{e}}, n$ and the plasma potential $V_{\mathrm{pl}}$ are now obtained from the measured quantities through ${ }^{9}$

$$
T_{\mathrm{e}}=\frac{e\left(V^{+}-V_{\mathrm{fl}}\right)}{\ln (3)}, \quad n=\frac{I}{e c_{s} A}, \quad V_{\mathrm{pl}}=V_{\mathrm{fl}}+\mu T_{\mathrm{e}} / e .
$$

In the following, we rederive relations (2) including two effects that can lead to important corrections. One is that due to stray capacitance to ground in the circuit, for instance capacitance in the coaxial cables connecting the pins. The other is due to an imperfect saturation of the ion current.

We begin with the measurement of the floating potential. Ideally, no current is drawn from the plasma. However, a finite leakage current exists due to stray capacitance (see Fig. 1). The potential of the probe, $V_{\mathrm{ff}}^{*}$, can thus deviate from the floating potential $V_{\mathrm{ff}}$. Using expression (A1) for the single LP current discussed in the Appendix, the current collected by the floating probe is given by

$$
\begin{aligned}
I= & I_{\mathrm{sat}}^{0}\left[1-\alpha\left(V_{\mathrm{fl}}^{*}-V_{\mathrm{fl}}\right)-\exp \left(\frac{V_{\mathrm{f}}^{*}-V_{\mathrm{fl}}}{T_{\mathrm{e} / e}}\right)\right] \\
& +C_{\mathrm{sh}}\left(\dot{V}_{\mathrm{pl}}-\dot{V}_{\mathrm{fl}}^{*}\right) .
\end{aligned}
$$

The parameter $\alpha$ accounts for the imperfect saturation of the ion current and $I_{\text {sat }}^{0}$ is the ion current extrapolated to the floating potential and given in Eq. (A2). Here, we have added a term due to the capacitance $C_{\text {sh }}$ of the sheath. This can be estimated as $C_{\mathrm{sh}} \approx \epsilon_{0} A / \lambda_{D}$ and is usually very small. ${ }^{6}$ For $T_{\mathrm{e}}=5 \mathrm{eV}, n=10^{16} \mathrm{~m}^{-3}$ and a probe surface $A$ of $10^{-5} \mathrm{~m}^{2}$ for example, we find that $C_{\mathrm{sh}} \approx 0.5 \mathrm{pF}$. A contribution of ion-polarization currents to Eq. (3) could also occur. ${ }^{6}$ For the present case where the scale length of electric fields around a probe tip are comparable or smaller than the ion Larmor radius, the estimate of such a contribution possibly requires kinetic studies and is not considered here.

The current in Eq. (3) equals the current flowing through the stray capacitance

$$
I=C_{\text {str }} \dot{V}_{\mathrm{fl}}^{*}
$$

We assume $C_{s t r} \gg C_{s h}$. Therefore, when we equate Eqs. (3) and (4), we neglect the sheath capacitance term in Eq. (3) with respect to the term in Eq. (4). If we further linearise the exponential term in (3) around $V_{\mathrm{fl}}$, neglect $\alpha$ with respect to $e / T_{\mathrm{e}}$ and arrange terms, we find

$$
V_{\mathrm{fl}}=V_{\mathrm{fl}}^{*}+\frac{T_{\mathrm{e}}}{e I_{\mathrm{sat}}^{0}} C_{\mathrm{str}} \dot{V}_{\mathrm{fl}}^{*} .
$$

We can set $R_{\mathrm{sh}}=T_{\mathrm{e}} / e I_{\text {sat }}^{0}$, the sheath resistance for a probe potential close to the floating potential. In cases where fluctuations of $R_{\mathrm{sh}}$ can be neglected, Eq. (5) is equivalent to an RC-circuit. In this case, the spectral components of $V_{\mathrm{fl}}$ and $V_{\mathrm{fl}}^{*}$ satisfy

$$
\hat{V}_{\mathrm{fl}}^{*}(f)=\frac{\hat{V}_{\mathrm{fl}}(f)}{1+2 \pi i f R_{\mathrm{sh}} C_{\mathrm{str}}} .
$$

We thus see that $\hat{V}_{\mathrm{fl}}^{*}(f)$ is reduced in amplitude and delayed with respect to $\hat{V}_{\mathrm{fl}}(f)$. Important phase errors occur already for fluctuations well below the cutoff frequency $f_{c}=1 /\left(2 \pi R_{\mathrm{sh}} C_{\mathrm{str}}\right)$. As an example, we assume again $T_{\mathrm{e}}$ $=5 \mathrm{eV}, n=10^{16} \mathrm{~m}^{-3}$ and $A=10^{-5} \mathrm{~m}^{2}$. In this case, using Eq. (A2) to express $I_{\text {sat }}^{0}$, we find that $R_{\mathrm{sh}} \approx 28 \mathrm{k} \Omega$. If we assume $C_{\mathrm{str}}=300 \mathrm{pF}$, typical for $1.5 \mathrm{~m}$ of a heat and vacuum resistent coaxial cable, we find $f_{\mathrm{c}} \approx 19 \mathrm{kHz}$. For fluctuations at $4 \mathrm{kHz}$, the phase error would already attain $0.2 \mathrm{rad}$. We 
further note that, since $R_{\mathrm{sh}} \propto \sqrt{T_{\mathrm{e}}} / n$, the sheath resistance can be significantly higher for lower values of plasma density.

In a similar way as we have done for the floating potential measurement, we apply current continuity to the double probe circuit (pins 2-4), neglect again sheath capacitance and assume $2 \cdot \exp \left(-e \Delta V / T_{\mathrm{e}}\right) \ll 1$. We find

$$
T_{\mathrm{e}}=\frac{e\left(V^{+}-V_{\mathrm{fl}}\right)}{\ln \left(3+\delta_{0}+\delta_{1}\right)},
$$

where we have defined $\delta_{0}$ and $\delta_{1}$ by

$$
\begin{aligned}
& \delta_{0}=\bar{\alpha}\left[2 \Delta V-3\left(V^{+}-V_{\mathrm{fl}}\right)\right], \\
& \delta_{1}=\tilde{\alpha}\left[2 \Delta V-3\left(V^{+}-V_{\mathrm{fl}}\right)\right]-\frac{3 C_{\mathrm{str}}}{I_{\mathrm{sat}}^{0}} \dot{V}^{+} .
\end{aligned}
$$

The overbar indicates time averages and the tilde fluctuating quantities such that $\tilde{\alpha}=\alpha-\bar{\alpha}$. Combining Eqs. (5) and (7) and expanding the logarithm, we find

$T_{\mathrm{e}}=\frac{e\left(V^{+}-V_{\mathrm{fl}}^{*}-\frac{T_{e}}{e I_{\mathrm{sat}}^{0}} C_{\mathrm{str}} \dot{V}_{\mathrm{fl}}^{*}\right)}{\ln \left(3+\delta_{0}\right)} \cdot\left[1-\frac{\delta_{1}}{\left(3+\delta_{0}\right) \ln \left(3+\delta_{0}\right)}\right]$.

With the same approximations, we finally find the expression for the current flowing in the circuit

$$
I=2 I_{\mathrm{sat}}^{0}\left[1+\alpha \Delta V-\alpha\left(V^{+}-V_{\mathrm{fl}}\right)\right]-2 C_{\mathrm{str}} \dot{V}^{+} .
$$

The density is then given by

$$
n=\frac{I+2 C_{\mathrm{str}} \dot{V}^{+}}{e c_{s} A_{\mathrm{eff}}^{0}\left(1+\epsilon_{0}+\epsilon_{1}\right)}
$$

with

$$
\begin{aligned}
& \epsilon_{0}=\bar{\alpha}\left[\Delta V-\left(V^{+}-V_{\mathrm{fl}}\right)\right], \\
& \epsilon_{1}=\tilde{\alpha}\left[\Delta V-\left(V^{+}-V_{\mathrm{fl}}\right)\right]
\end{aligned}
$$

and $A_{\text {eff }}^{0}$ the ion sheath surface at floating potential (see discussion in the Appendix).

Equations (10) and (12) are the expressions of the modified triple probe method for $T_{\mathrm{e}}$ and $n$ with effects of stray capacitance and ion sheath expansion included. Equation (5) gives further the expression for $V_{\mathrm{fl}}$ needed for the evaluation of the plasma potential through $V_{\mathrm{pl}}=V_{\mathrm{fl}}+\mu T_{\mathrm{e}} / e$. In the limit of $C_{\text {str }}$ and $\alpha \rightarrow 0$, these expressions agree with those in (2). In this work, we will take into account corrections due to $\bar{\alpha}$ and neglect terms proportional to $\tilde{\alpha}$, as discussed in Sec. V B. As far as stray capacitance effects are concerned, one might want to correct numerically for these effects in Eqs. (5), (10), and (12). However, these depend on plasma parameters and involve nonlinear terms and time derivatives. It seems therefore better to keep $C_{\text {str }}$ sufficiently low. Often, coaxial cables of $1 \mathrm{~m}$ or more are required between probe and electronics. In the next section, we show how stray capacitance introduced by this can effectively be reduced.

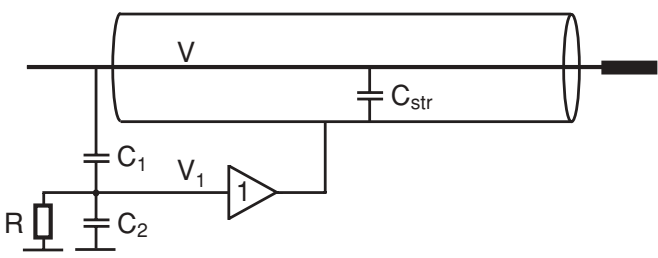

FIG. 2. Guarding circuit to reduce the capacitance between the inner conductor and the shield of a coaxial cable. "1" represents a unity gain voltage follower.

\section{A NOVEL GUARDING CIRCUIT TO REDUCE STRAY CAPACITANCE}

In this section, we describe a novel guarding circuit that allows a strong reduction of the stray capacitance. An idealized scheme of this circuit is shown in Fig. 2 . The basic idea is to drive the shield of the coaxial cable to follow potential fluctuations in the inner conductor. This is done by the unity gain voltage follower in Fig. 2. It allows reducing the current needed to charge the capacitance $C_{\text {str }}$ and thus reduces the effective capacitance in the circuit, while still keeping the shielding properties of the coaxial cable with respect to external noise. Relatively large dc potential values can arise in the circuit (e.g., for tips on $V^{-}$). These can exceed the voltage limits of the voltage follower in the circuit. Therefore, the system is ac coupled through the potential divider including $C_{1}, C_{2}$, and $R$. This circuit thus reduces stray currents at relatively high frequencies where they are important. At low frequencies, stray currents are even increased due to the additional capacitance $C_{1}$. Although the new circuit is not purely capacitive anymore, we still represent it by an effective, frequency dependent capacitance $C_{\text {eff }}(f)$. We find

$$
C_{\mathrm{eff}}(f)=\left(C_{\mathrm{str}}+C_{1}\right) \frac{1+2 \pi i f R C_{2}}{1+2 \pi i f R\left(C_{1}+C_{2}\right)},
$$

such that the leakage currents $I_{\text {leak }}$ that were simply given by terms of the form $C_{\text {str }} \dot{V}$ in the previous section now become

$$
I_{\text {leak }}=F T^{-1}\left[2 \pi i f C_{\text {eff }}(f) \hat{V}(f)\right] .
$$

Here $F T^{-1}$ is the inverse Fourier transform. The module $\left|C_{\text {eff }}\right|$ is a decreasing function of $f$, with the following properties

$$
\begin{aligned}
& C_{\mathrm{eff}}(f \rightarrow 0)=C_{\mathrm{str}}+C_{1}, \\
& C_{\mathrm{eff}}(f \rightarrow \infty)=\left(C_{\mathrm{str}}+C_{1}\right) \frac{C_{2}}{C_{1}+C_{2}}, \\
& \frac{\left|C_{\mathrm{eff}}(f)\right|}{C_{\mathrm{str}}}<\frac{1}{2} \text { for } f>f_{1 / 2} \approx \frac{C_{1}+C_{\mathrm{str}}}{\pi R C_{1} C_{\mathrm{str}}} .
\end{aligned}
$$

For the estimate of $f_{1 / 2}$, we have assumed $C_{2}$ $\ll C_{\text {str }}, C_{1}$. The reduction of $\left|C_{\text {eff }}(f)\right|$ with respect to $C_{\text {str }}$ becomes thus effective only for frequencies $\gtrsim f_{1 / 2}$. Therefore, $f_{1 / 2}$ should be smaller than the frequencies that give rise to important stray currents. As $f_{1 / 2} \propto 1 / R$ [Eq. (19)], this can be controlled by the value of the resistance $R$ in the circuit. 
However, the value of $R$ should not be chosen too large, otherwise the dc decoupling of the circuit becomes less effective. As the plasma is turned on, the dc part of the potential $V$ of the inner conductor can change strongly. The potential $V_{1}$ of the shield follows this change and can thus exceed the potential limits of the amplifier. The dc part of $V_{1}$ relaxes to 0 at a characteristic time of $\tau=R\left(C_{1}+C_{2}\right) \approx R C_{1}$. This characteristic time should be kept short compared to the length of the plasma phase.

\section{TRIPLE PROBE AND GUARDING CIRCUITRY CONSTRUCTION}

In Fig. 3 , we show a photograph and a scheme of the probe head. The five tips are made of stainless steel wires of $0.7 \mathrm{~mm}$ diameter. The wires protrude by $6 \mathrm{~mm}$ from the ceramic tubes of $6 \mathrm{~cm}$ in length that separate the tips from the boron nitride $(\mathrm{BN})$ casing. A replacable piece made out of Vespel ${ }^{\circledR}$ allows a good alignment of the tips as well as a variable spacing between adjacent tips in the range of $2.5-6 \mathrm{~mm}$. For this work, a tip spacing of $4 \mathrm{~mm}$ is used. For $T_{\mathrm{e}}=5 \mathrm{eV}$ and $n=10^{16} \mathrm{~m}^{-3}$, this corresponds to a gap between tips of $\approx 20$ Debye lengths.

The probe is installed on a 1D movable system that allows moving the probe over the whole radial range of the TORPEX vacuum vessel. The probe tips are aligned perpendicular to the magnetic field and in the vertical direction. At least for the ideal interchange regime in TORPEX (Refs. 14 and 15) where plasma profiles are slab-like, this corresponds to the direction perpendicular to the gradients in the plasma profiles.

Inside the BN casing, the stainless steel wires are connected to coaxial cables that transport the signals to the electronics right behind the vacuum-feedthrough. Coaxial cables with an outer diameter of $1.4 \mathrm{~mm}$ and a low capacitance of $50 \mathrm{pFm}^{-1}$ are used. They have a length of approximately
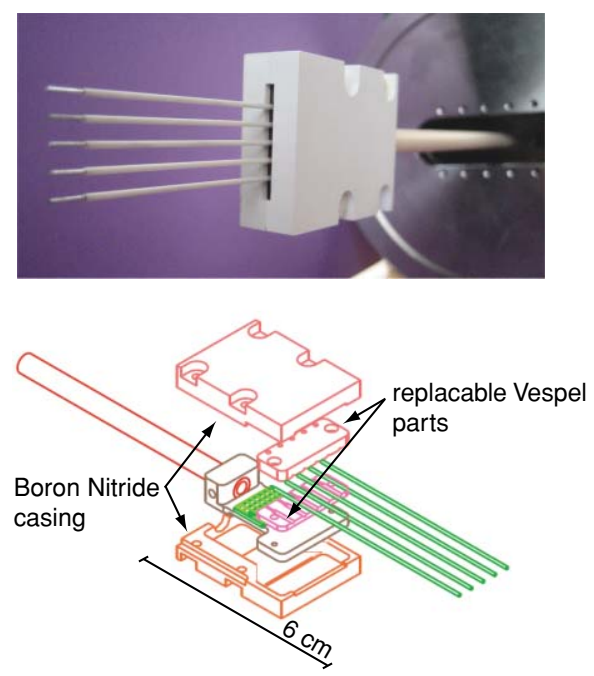

FIG. 3. (Color online) Top: photograph of the probe head showing the five probe tips, the BN casing, and (in the background) a stainless steel flange. Bottom: scheme showing the interior of the probe. A central part made out of Vespel ${ }^{\circledR}$ is holding the probe head. Replacable Vespel ${ }^{\circledR}$ parts allow controlling alignment and spacing of the five probe tips. The interior is protected by a BN casing.
$1.2 \mathrm{~m}$ resulting in a stray capacitance to ground for each individual channel of $\approx 70 \mathrm{pF}$. To further reduce this capacitance, the circuit described in Sec. III is applied to each channel. The values $C_{1}=1200 \mathrm{pF}, C_{2}=8 \mathrm{pF}$ and $R=10 \mathrm{M} \Omega$ are chosen. For stability reasons, a resistance of $15 \mathrm{k} \Omega$ is added in series to $C_{1}$, with very little effect on $\left|C_{\text {eff }}\right|$ for the frequency range of interest. This allows us to reduce the stray capacitance of each channel to approximately $20 \mathrm{pF}$, including compensated potential dividers for the potential measurements. Finally, the current signal in the double probe circuit is measured over a $10 \Omega$ resistance and numerically corrected for instrumental transfer function.

\section{EXPERIMENTAL RESULTS}

The probe is tested in the simple magnetized toroidal device TORPEX (Ref. 10) (major radius of $1 \mathrm{~m}$ and minor radius of $0.2 \mathrm{~m}$ ) using hydrogen plasmas produced and sustained by means of microwaves in the electron cyclotron range of frequencies. A relatively small vertical magnetic field component $B_{z}$ is superimposed on a dominant toroidal component of $B_{\text {tor }} \approx 0.08 \mathrm{~T}$. This leads to helical field lines that intercept the vacuum vessel at the bottom and the top. The nature of the dominant instability can be controlled by the strength of $B_{z} \cdot{ }^{14,15}$ In this work, we set $B_{z}=2.4 \mathrm{mT}$, which falls in the regime dominated by an ideal interchange instability. ${ }^{14,16}$ This configuration has been studied in detail previously. ${ }^{16,17}$ A relatively low injected microwave power $(300 \mathrm{~W})$ results in vertically elongated, slab-like profiles that peak on the highfield side, i.e., on the inner side of the device cross section. An interchange wave develops on the low field side region, where pressure gradients and magnetic field gradients are collinear. The field lines perform approximately two toroidal turns inside the vessel and the wave grows on the longest allowed vertical scale, which results in a vertical wave length of $\lambda \approx 20 \mathrm{~cm}$. The mode propagates upwards due to a background $\mathrm{E} \times \mathrm{B}$ flow with a phase velocity of approximately $800 \mathrm{~ms}^{-1}$ resulting in a measured frequency of $\approx 3-4 \mathrm{kHz}$.

In the following, this plasma is diagnosed with the new probe, operated either in the tripe probe set-up or in swept mode. Measurements are possible over the whole radial range at midplane. Except for the analysis in Fig. 8, we always use the 5-tip scheme for triple probe measurements.

\section{A. Consistency tests}

After installation, the probe tips have been biased to $-40 \mathrm{~V}$ with respect to device ground during plasma phases of $\sim 1$ min to sputter clean them. This has allowed us to remove hysteresis effects observed on the $I-V$ characteristics due to probe surface impurity contamination.

A necessary condition for reliable measurements with the triple probe is that all probe tips show similar plasma response. In particular, they should provide similar measurements of time averaged plasma parameters when operated in swept mode. To test this, a sawtooth potential sweep at $330 \mathrm{~Hz}$ between $-40 \mathrm{~V}$ and $+20 \mathrm{~V}$ is applied simultaneously to all five probe tips. The $I-V$ characteristics, averaged over many sweeping cycles, is obtained and fitted with Eq. (A1) to 

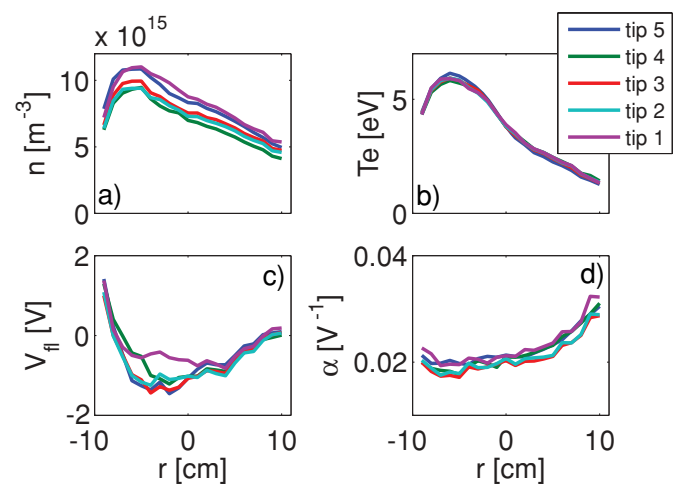

FIG. 4. (Color online) Radial profiles of time average (a) density, (b) electron temperature, (c) floating potential, and (d) the parameter $\alpha$ for the five probe tips obtained by slowly sweeping the probe voltages.

determine $n, T_{\mathrm{e}}, V_{\mathrm{fl}}$, and the parameter $\alpha$. The radial profiles of these parameters are shown in Fig. 4. We see that tips 1 and 5 measure densities about $\approx 15 \%$ higher than the tips in between. This could be due to a slight overlap of the probe sheaths and might be further investigate by using different distances between the probes in the future. Some differences are also observed in the floating potential measurements. Nevertheless, the profiles of the different probe tips are in good agreement.

Next, we increase slightly the sweeping range $(-40$ to $+40 \mathrm{~V}$ ) to evaluate $V_{\mathrm{pl}}$ from the knee of the electron current characteristics and to determine the factor $\mu$ that links $V_{\mathrm{pl}}, V_{\mathrm{fl}}$, and $T_{\mathrm{e}}$. Very good agreement between the profiles of $V_{\mathrm{pl}}$ and $V_{\mathrm{fl}}+\mu T_{e} / e$ is found for $\mu=3.1 \pm 0.2$.

We now use the probe in the (five tip) triple probe configuration. First, we want to make sure that stray capacitances have been reduced sufficiently for the present plasma configuration to have only negligible effects on the measurements. For this, we determine the parameters $T_{\mathrm{e}}, n$, and $V_{\mathrm{fl}}$ from Eqs. (5), (10), and (12), with and without realistic estimates of the $C_{\text {str }}$-terms. The time traces of the evaluated quantities with and without these corrections, labeled, e.g., as $n^{\text {corr }}$ and $n$, look very similar. To quantify this for the whole radial range, we plot in Fig. 5 the average difference between corrected and noncorrected quantities, normalized to the standard deviation of the signals, as well as the mutual phase differences. We find indeed that corrections due to stray capacitances are weak here. The average absolute difference is smaller than $10 \%$ of the signals standard deviation and phase errors are below $0.08 \mathrm{rad}$. These tests will need to be repeated in TORPEX for different plasmas, especially for plasmas with higher fluctuation frequencies or lower densities.

We present in the next two sections cross-checks of triple probe measurements with other LP-techniques and measurements achieved only with the new probe.

\section{B. Cross-checks with other LP-based techniques}

In this section, we compare measurements obtained with the triple probe with results from other LP-based techniques. We start with time-averaged profiles. In Fig. 6, we plot the time-averages of $n, T_{\mathrm{e}}$, and $V_{\mathrm{fl}}$ obtained with the triple probe

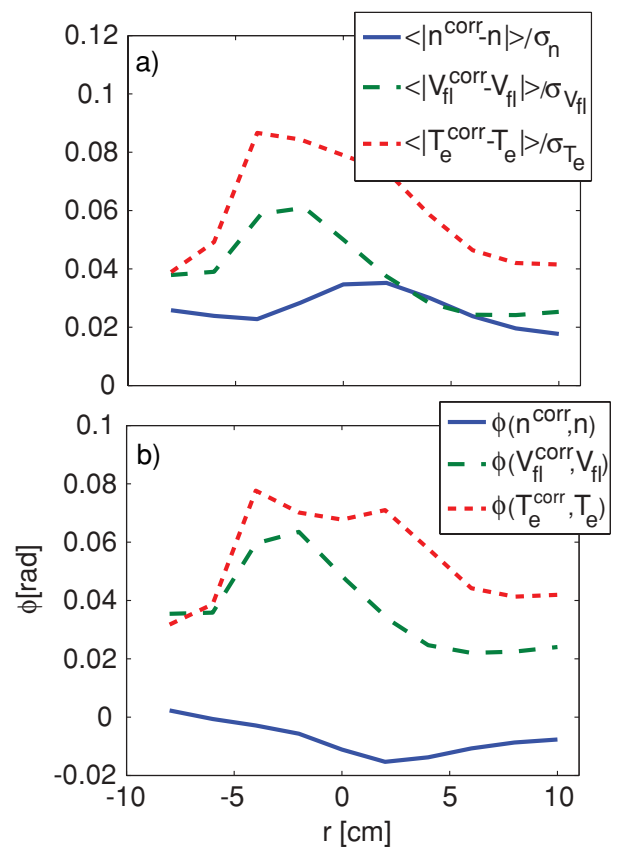

FIG. 5. (Color online) Study of the effects of the residual stray capacitances on the measurements. $n^{\text {corr }}, T_{\mathrm{e}}^{\text {corr }}$, and $V_{\mathrm{ff}}{ }^{\text {corr }}$ are obtained from Eqs. (5), (10), and (12) including realistic stray capacitances while $n, T_{\mathrm{e}}$, and $V_{\mathrm{fl}}$ are obtained with $C_{\text {str }}=0$. In (a), we compare the average difference between corrected and noncorrected signals with the signals standard deviations. In (b), we deduce the mutual phase shifts, evaluated by weighting the frequency dependent phase shift with the power spectral density of the noncorrected signals.

as well as from analyzing the $I-V$ characteristic as discussed in the previous section. We neglect $C_{\text {str }}$ and $\tilde{\alpha}$ in Eqs. (5), (10), and (12). If we further set $\bar{\alpha}=0$, we obtain the thick dashed profiles in Fig. 6, which deviate significantly from the profiles obtained from sweeping the five probe tips (thin lines). Including the values for $\bar{\alpha}$ of Fig. 4(d), however, leads to a satisfactory agreement for $n$ and $T_{\mathrm{e}}$ (thick solid profiles).

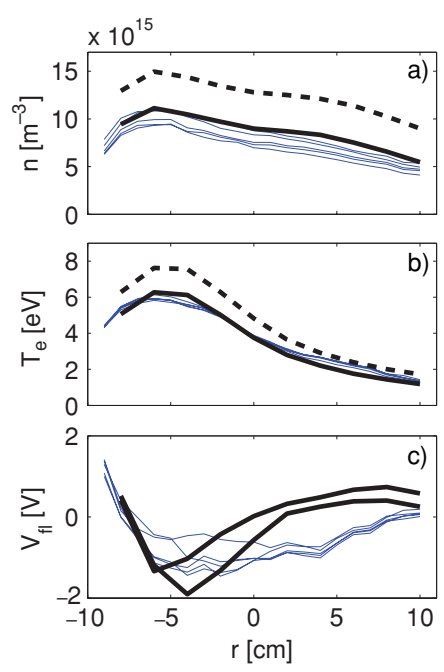

FIG. 6. (Color online) Time-averaged profiles of (a) density, (b) electron temperature, and (c) floating potential. Thin blue lines show the profiles obtained from sweeping the probes. Thick dashed lines in (a) and (b) show the profiles obtained with the triple probe with $\bar{\alpha}=0$, solid lines the ones including $\bar{\alpha}$-corrections. The two thick lines in (c) are the time-averages for the outer two probe tips (tips 1 and 5) that are operated in floating potential. 
There are some differences in the profiles of $V_{\mathrm{fl}}$ for the two techniques, Fig. 6(c). These are not entirely understood. We note, however, that these differences are relatively small and the two techniques result in very similar profiles of plasma potential $V_{\mathrm{pl}}=V_{\mathrm{fl}}+\mu T_{\mathrm{e}} / e$.

Since we are sweeping the probes at a frequency well below that of the dominant fluctuations in the plasma, we can not obtain the time evolution of $n, T_{\mathrm{e}}$, and $V_{\mathrm{fl}}$ and compare them with triple probe measurements. We can nevertheless obtain the average evolution of the interchange wave using a modified conditional sampling method. This technique is explained in details elsewhere ${ }^{18}$ and can be summarized as follows: a fixed reference probe in the mode region measures the ion saturation current $I_{\mathrm{sat}}$ and one or several movable probes are operated in swept mode. In the $I_{\text {sat }}$ time trace, we are detecting positive wave crests as they move in front of the probe. This can be done efficiently by first filtering the signal around the mode frequency and then selecting local maxima. To each time a positive wave crest is detected corresponds one measured current value $I$ and one applied voltage value $V$ on each swept probe. Since the mode frequency is not correlated with the voltage sweep applied to the probes, the ensemble of currents and voltages gives an $I-V$ characteristics which can be fitted to estimate plasma parameters. This exercise is repeated at different time lags $\tau$ around the time of the detection of the mode structure. This can be repeated for different locations of the swept probes to reconstruct the 2D conditionally averaged evolution of the mode.

In Fig. 7, we show the results of this analysis for the triple probe positioned at $r=-1 \mathrm{~cm}$, which corresponds to the center of the mode region. A reference signal in the mode region and toroidally separated by $\approx 35^{\circ}$ from the triple probe is used. First, a voltage sweep is applied to the five probe tips and the modified conditional sampling method discussed above is applied. The result for the central tip is shown in Fig. 7, blue solid lines. We see that the amplitude of the fluctuations decreases as we move away from

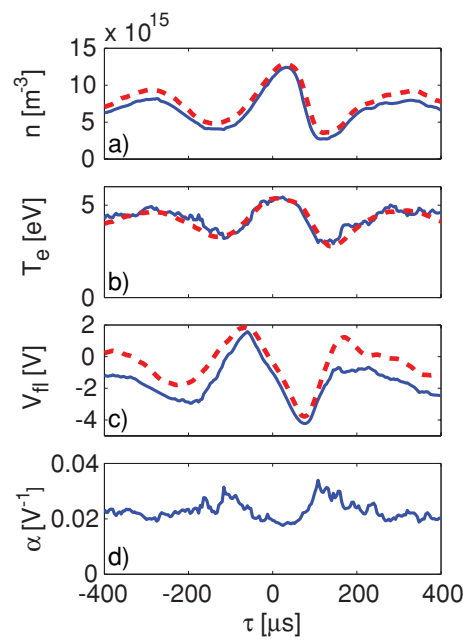

FIG. 7. (Color online) Conditionally averaged time traces of (a) density, (b) electron temperature (c) floating potential, and (d) parameter $\alpha$ in the mode region at $r=-1 \mathrm{~cm}$. Blue solid lines are obtained with a modified conditional sampling method from swept probes (Ref. 18). Red dashed lines are obtained with standard conditional sampling applied to triple probe data.

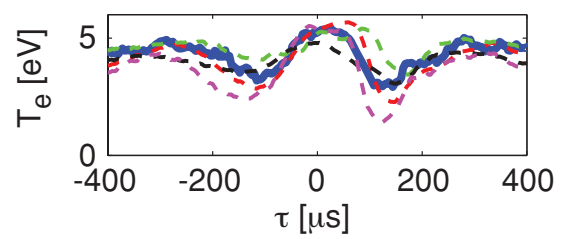

FIG. 8. (Color online) Comparison of the conditionally averaged time traces of $T_{\mathrm{e}}$ obtained from the swept probe (thick blue line) with results from the standard, three-tip triple probe. Different colors correspond to the following tip order (from bottom to top): red: $\left(V_{\mathrm{ff}}, V^{+}, V^{-}\right)$, green: $\left(V^{+}, V^{-}, V_{\mathrm{ff}}\right)$, black: $\left(V^{-}, V^{+}, V_{\mathrm{fl}}\right)$, violet: $\left(V_{\mathrm{fl}}, V^{-}, V^{+}\right)$.

$\tau=0$, which is a common feature of conditional sampling. Then, we repeat the measurements with the same reference signal, using the new probe in the triple probe scheme. Applying standard conditional sampling, we obtain the red dashed curves in Fig. 7. We find very satisfactory agreement between the two techniques in terms of fluctuation amplitudes, phase shifts and absolute values. An offset in the floating potential is observed, similarly to the time-average profiles in Fig. 6(c). A similarly good agreement is found at the the other radial locations where this analysis was performed, i.e., for $r=-6 \mathrm{~cm}$, $r=-3 \mathrm{~cm}$, and $r=3 \mathrm{~cm}$.

In Fig. 7(d), we show the conditionally sampled time trace of $\alpha$. $\alpha$ fluctuates and has a phase shift of $\pi$ with respect to density and temperature. To include $\alpha$-fluctuations in the analysis of triple probe data [see Eqs. (10) and (12)], a study of the dependence of $\alpha$ on plasma parameters would be necessary, but this is beyond the scope of the present work. Another possibility is to use different values of $\Delta V$ for the triple probe and thus change the influence of the $\alpha$-terms in Eqs. (10) and (12). We have done this for $\Delta V=24,36$, and $48 \mathrm{~V}$, but no significant differences were found as long as corrections due to $\bar{\alpha}$ were included. We therefore neglect the $\tilde{\alpha}$-terms in Eqs. (10) and (12).

Finally, to evaluate the effect of phase delay errors, we repeat the conditional sampling analysis for the configurations in the standard, 3-tip triple probe scheme that are possible by opening or closing the switches A and B (see Fig. 1). This allows for the following combinations: $\left(V_{\mathrm{ff}}, V^{+}, V^{-}\right)$, $\left(V^{+}, V^{-}, V_{\mathrm{ff}}\right),\left(V^{-}, V^{+}, V_{\mathrm{ff}}\right)$, and $\left(V_{\mathrm{ff}}, V^{-}, V^{+}\right)$. The results of this for $T_{\mathrm{e}}$ are shown in Fig. 8 and compared with the result from the swept probe (thick solid blue line). We observe similar time-average values, but considerable differences in both phase and amplitude of fluctuations for all four cases. This clearly motivates the use of the modified, five-tip method.

\section{Fluctuation measurements with the triple probe}

After the successful cross-check of the triple probe measurements with other LP techniques, we present now the main results obtained with this probe in the plasma under investigation. The main properties of the fluctuations of $n, I_{\text {sat }}, T_{\mathrm{e}}$, $V_{\mathrm{fl}}$, and $V_{\mathrm{pl}}$ are summarized in Fig. 9. In (a), we show rawdata time traces at a radial position of $r=-2 \mathrm{~cm}$ for approximately three periods of the interchange wave. In (b), we compare the relative fluctuation levels for the positive-definite 

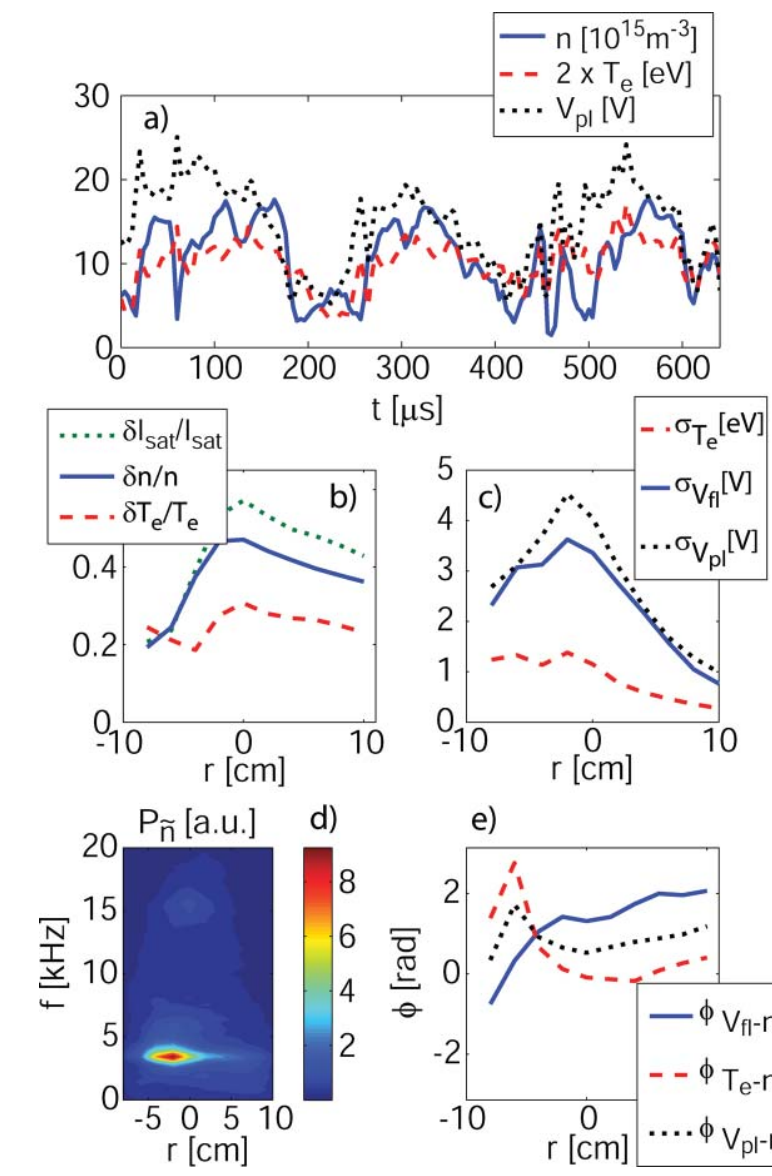

e)

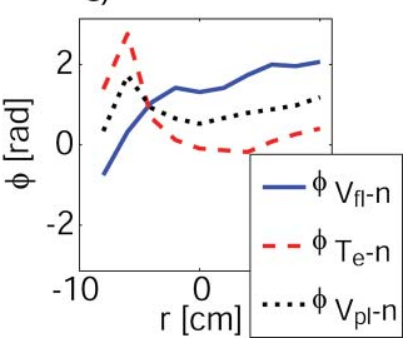

FIG. 9. (Color online) Fluctuation measurements with the modified, 5-tip triple probe: (a) time traces of $n, T_{\mathrm{e}}$, and $V_{\mathrm{pl}}$; (b) relative fluctuation levels of $I_{\mathrm{sat}}, n$, and $T_{\mathrm{e}}$; (c) standard deviation of $T_{\mathrm{e}}, V_{\mathrm{fl}}$, and $V_{\mathrm{pl}} ;(\mathrm{d})$ power spectral density of $\tilde{n}$ as a function of $r$; (e) phase shifts between $V_{\mathrm{fl}}, T_{\mathrm{e}}, V_{\mathrm{pl}}$ and $n$ in the frequency range $[2.4,4.4] \mathrm{kHz}$ of the dominant mode.

quantities $I_{\text {sat }}, n$, and $T_{\mathrm{e}}$. The fluctuation level is defined as the signal standard deviation over its mean value. We find the commonly observed large fluctuation levels, with $\delta T_{\mathrm{e}} / T_{\mathrm{e}}$ roughly two thirds of $\delta n / n$. In (c), we compare fluctuations in $T_{\mathrm{e}}, V_{\mathrm{fl}}$, and $V_{\mathrm{pl}}$ by plotting the signal standard deviations. We find that $\sigma_{V_{\mathrm{fl}}} \approx 3 \cdot \sigma_{T_{e}} / e$. This shows clearly that temperature fluctuations should be taken into account when evaluating $V_{\mathrm{pl}}$ from $V_{\mathrm{fl}}+\mu T_{\mathrm{e}} / e$. In Fig. 9(d), we plot the power spectral density of $\tilde{n}$. The main instability at a frequency of $3.4 \pm 0.5 \mathrm{kHz}$ and its radial extension are apparent. A much weaker mode at a frequency of $15.5 \pm 1.5 \mathrm{kHz}$ is also present and can be identified as the first harmonic of the dominant mode, despite the fact that its frequency is higher by a factor 4-5.

Focusing on fluctuations related with the dominant instability, we show in Fig. 9(e) radial profiles of the phaseshift between $T_{\mathrm{e}}, V_{\mathrm{fl}}$, and $V_{\mathrm{pl}}$ with respect to density, averaged over the frequency range $3.4 \pm 1 \mathrm{kHz}$. We find that the phase shift between temperature and density is close to zero for $r \geq-2 \mathrm{~cm}$. It increases for smaller values of $r$ and is close to $\pi$ at $r=-6 \mathrm{~cm}$. This feature is consistent with the conditionally averaged time traces for $r=-3 \mathrm{~cm}$ and $r=-6 \mathrm{~cm}$ mentioned in the previous section. We further observe a phase shift between plasma potential and density that is significantly smaller than that between floating potential and density. The sign of the phase shift between $V_{\mathrm{pl}}$ and $n$ is consistent with that expected for an interchange wave. However, as noted earlier, ${ }^{18}$ the phase shift is in the range $0.5-0.9 \mathrm{rad}\left(30^{\circ}-50^{\circ}\right)$ in the mode region, clearly below the $90^{\circ}$ phase shift expected from linear theory.

We turn now to the evaluation of particle transport with triple probe measurements. More specifically, we focus on the time-average radial particle flux $\left\langle\Gamma_{r}\right\rangle$. In the present case where magnetic fluctuations can be neglected, we can write $\left\langle\Gamma_{r}\right\rangle=\left\langle n v_{r}\right\rangle$ with $v_{r}=-E_{z} / B$ the radial $\mathbf{E} \times \mathbf{B}$ velocity. The minus sign in the expression for $v_{r}$ stems from the direction of the magnetic field. In the present experiments, $\mathbf{B}$ is directed in the counter-clockwise direction if the torus is seen from the top, such that a positive radial particle flux corresponds to a negative vertical electric field $E_{z}$. Setting $n=\bar{n}+\tilde{n}$ and $v_{r}=\bar{v}_{r}+\tilde{v}_{r}$, we can decompose the flux in a steady state component and a contribution due to the fluctuations in the plasma

$$
\left\langle\Gamma_{r}\right\rangle=\bar{n} \bar{v}_{r}+\left\langle\tilde{n} \tilde{v}_{r}\right\rangle
$$

We discuss the first term on the right-hand side quickly at the end of this section and focus now on the term caused by fluctuations of $n$ and $v_{r}$. A difficulty arises in the estimation of $\tilde{v}_{r}$ as it requires the determination of vertical electric field fluctuations, while the triple probe provides only one local measurement of the plasma potential. This difficulty can be overcome in cases where fluctuations satisfy a dispersion relation, i.e., when we can write $k_{z}=k_{z}(f)$. In this case, one finds ${ }^{19}$

$$
\left\langle\tilde{n} \tilde{v}_{r}\right\rangle=\frac{2}{B} \int_{0}^{\infty} \mathrm{d} f k_{z} \sqrt{P_{\tilde{V}_{\mathrm{pl}}} P_{\tilde{n}}} \gamma \sin (\phi)
$$

with $\gamma=\gamma(f)$ and $\phi=\phi(f)$ being the coherence and phase angle between $\tilde{V}_{\mathrm{pl}}$ and $\tilde{n}$ and $P_{\tilde{V}_{\mathrm{pl}}}, P_{\tilde{n}}$ their power spectral densities. The wave number $k_{z}(f)$ can be estimated experimentally from two-point correlation ${ }^{13}$ of the $V_{\mathrm{fl}}$-signals acquired at tip 1 and 5 of the probe (see Fig. 1) and one local measurement of $V_{\mathrm{pl}}$ is now sufficient to determine $\left\langle\tilde{n} \tilde{v}_{r}\right\rangle$.

The flux $\left\langle\tilde{n} \tilde{v}_{r}\right\rangle$ is linear in $\tilde{V}_{\mathrm{pl}}$ and we can thus separate Eq. (21) in a contribution from $\tilde{V}_{\text {fl }}$ and a contribution from $\mu \tilde{T}_{\mathrm{e}} / e$. These two contributions are shown in Fig. 10 respectively as green diamonds and red squares. As expected from the positive phase shift between $\tilde{V}_{\mathrm{fl}}$ and $\tilde{n}$ in Fig. 9, floating potential fluctuations drive a positive radial flux, while the time-average contribution from electron temperature fluctuations is weak. The error bars for these curves take into account uncertainties in the phase of the $V_{\mathrm{fl}}$ measurements (Fig. 5), in $\mu$, and in $k_{z}(f)$, as well as a reduction of the fluctuation amplitude of $V_{\mathrm{fl}}=\left(V_{\mathrm{fl} 1}+V_{\mathrm{fl} 5}\right) / 2$ introduced by averaging the signals from tips 1 and 5. The latter two dominate the error bars for transport due to temperature fluctuations. The error bars of the floating potential driven transport are dominated by $\delta k_{z}(f)$ alone, which we take as the standard deviation of the wavenumber-frequency (statistical) spectrum. We note that we have included all fluctuations with $f \leq 50 \mathrm{kHz}$ in the transport calculation. Significantly smaller error bars than in the present case are obtained when calculating the 


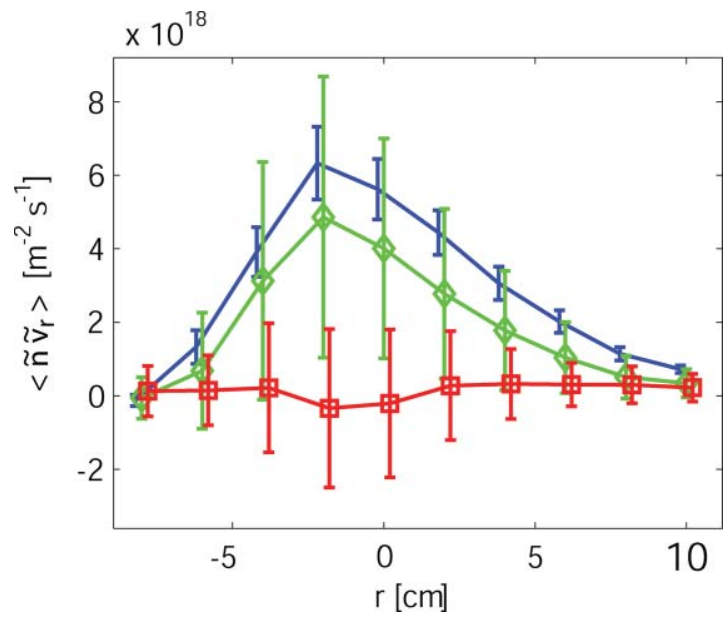

FIG. 10. (Color online) Radial particle flux associated with fluctuations in $V_{\mathrm{ff}}$ (diamonds) and $T_{\mathrm{e}}$ (squares) calculated assuming a dispersion relation, Eq. (21). The blue profile is the radial fluctuation induced flux obtained from Eq. (22). For clarity, the three profiles have been slightly displaced radially such that error bars do not lie on top of each other.

contribution from a coherent part of the spectrum only (here the $3.4 \mathrm{kHz}$ mode).

As temperature fluctuations are found to contribute weakly to the radial particle transport, it seems more direct to evaluate $\left\langle\tilde{n} \tilde{v}_{r}\right\rangle$ from electric field fluctuations deduced from gradients in the floating potential. We thus set

$$
\left\langle\tilde{n} \tilde{v}_{r}\right\rangle=\left\langle\tilde{n} \frac{\tilde{V}_{\mathrm{fl} 5}-\tilde{V}_{\mathrm{fl} 1}}{d_{\mathrm{tip}} B}\right\rangle
$$

with $d_{\text {tip }}=1.6 \mathrm{~cm}$ the spacing between tip 1 and 5 . Results are shown as a blue solid line in Fig. 10. Error bars are deduced from uncertainties in the tip spacing and the phase of the floating potential signals as well as differences in the fluctuation level of $\tilde{V}_{\mathrm{fl} 1}$ and $\tilde{V}_{\mathrm{fl} 5}$. In the mode region, we find reasonable agreement between transport driven by $\tilde{V}_{\mathrm{fl}}$ evaluated in this way and that through Eq. (21). A relative difference of $\approx 20 \%$ between the two is found in the central region of the mode at $r=-2 \mathrm{~cm}$. The relative difference between the two increases monotonically up to $\approx 55 \%$ at $r=10 \mathrm{~cm}$. In that region, cross-field transport is dominated by the propagation of turbulent structures (blobs) that do not satisfy a dispersion relation and Eq. (21) cannot properly account for their transport. ${ }^{20,21}$

For the present case where the phase between electron temperature and density is small, the calculation of $\left\langle\tilde{n} \tilde{v}_{r}\right\rangle$ with the simple formula (22) seems more accurate than that with Eq. (21), which relies on the existence of a dispersion relation. We note, however, that the interpretation of the two terms on the right hand side of Eq. (20) as the transport due to the background and due to turbulence, respectively, is not necessarily meaningful if velocity perturbations due to turbulence are not zero on average, as can be the case if turbulence is vertically asymmetric. In such a case, other definitions of the turbulent flux, such as that associated with blobs as defined in Refs. 20 and 21 for example, are more appropriate. Further, to calculate the total radial particle transport $\left\langle\Gamma_{r}\right\rangle$, the measurement of $\bar{n} \bar{v}_{r}$ is required as well. We do not address this issue here but simply remark that a difference of $1 \mathrm{~V}$ between the time- average plasma potential at tip 1 and 5, which at $r=-2 \mathrm{~cm}$ is less than $10 \%$ of $V_{\mathrm{pl}}$ and thus difficult to measure, would already result in $\left|\bar{n} \bar{v}_{r}\right| \sim\left|\left\langle\tilde{n} \tilde{v}_{r}\right\rangle\right|$.

\section{SUMMARY AND CONCLUSIONS}

We have described the construction and use of the triple Langmuir probe $^{8}$ in toroidal, magnetized plasmas of the TORPEX device. Difficulties with this method in obtaining reliable time-resolved measurements of plasma density, electron temperature, floating, and plasma potential have been discussed and overcome: the probe tips are aligned perpendicularly to the gradients in the background profiles and on separate field lines to reduce shadowing effects. A symmetric, 5-tip scheme ${ }^{9}$ is used to reduce errors caused by gradients in the fluctuating parameters. The dependence of the ion saturation current on probe voltage is accounted for in an approximate way. Finally, the effect of stray capacitance on the measurement bandwidth has been discussed in detail. Bandwidth limitations become more serious as density and probe surface decrease. A new guarding circuit is described to reduce the main source of stray capacitance in the present work, that of the coaxial cables between probe tips and electronics.

The probe has been tested in a plasma characterized by interchange-driven turbulence in TORPEX. Time-averaged and time-dependent, conditionally averaged measurements of density, electron temperature and floating potential have been obtained in two ways, with the triple probe method and from applying a voltage sweep to the probe tips. Very satisfactory agreement is found between the two methods in terms of absolute values, fluctuation levels and phase shifts of the different quantities. The triple probe measurements give detailed insights on the interchange dynamics. The phase shift between density and electron temperature is close to zero over most of the radial range. Density and plasma potential are shifted by $30^{\circ}-50^{\circ}$, less than the $90^{\circ}$ expected from linear calculations. The fluctuation level of electron temperature is about two third of that in density and important in the evaluation of plasma potential from the relation $V_{\mathrm{pl}}=$ $V_{\mathrm{fl}}+\mu T_{\mathrm{e}} / e$. Nevertheless, it is concluded that in the present case of weak phase shifts between density and electron temperature, the time averaged fluctuation induced particle flux can be reliably deduced with Eq. (22) from floating potential measurements.

Future application of the new probe in TORPEX will be to extend the set of observables for the TORPEX turbulence code validation project ${ }^{22,23}$ and to obtain detailed measurements of quantities related with turbulent structures (blobs) to improve our understanding of their motion and control. ${ }^{24}$ This will require further studies. Despite the good agreement between triple probe and single LP measurements reported in this work, there is clearly a lower limit in the scale-length of fluctuations that can be properly resolved with the triple probe method. Blobs for example have a typical density scale-length of $\approx 2 \mathrm{~cm}$ (Ref. 24) and already constitute a challenge for the triple probe. Such difficulties will be further studied with comparisons between different techniques (e.g., conditionally averaged triple probe and 
single LP measurements of blobs), by varying the tip spacing of the triple probe or by using a synthetic triple probe in simulations of TORPEX plasmas..$^{15,22}$

\section{ACKNOWLEDGMENTS}

The authors thank P. Gorgerat for the probe technical drawings and P. Lavanchy for the assembly of the electronics and assistance for its characterization, and acknowledge many useful discussions with A. Howling, B. Labit and M. Podestà. This work is partially supported by the Fonds National Suisse de la Recherche Scientifique.

\section{APPENDIX: SINGLE LANGMUIR PROBES IN TORPEX PLASMAS}

In TORPEX, Langmuir probe measurements are interpreted by the following relation between the current $I$ collected by the probe and the probe potential $V_{\mathrm{pr}}$

$$
I\left(V_{\mathrm{pr}}\right)=I_{\mathrm{sat}}^{0}\left[1-\alpha\left(V_{\mathrm{pr}}-V_{\mathrm{fl}}\right)-\exp \left(\frac{V_{\mathrm{pr}}-V_{\mathrm{fr}}}{T_{\mathrm{e}} / \mathrm{e}}\right)\right] .
$$

The first two terms on the right-hand side represent the ion current to the probe. The imperfect saturation of the ion current at low $V_{\mathrm{pr}}$ due to sheath expansion is approximated by a linear relation. The third term on the right hand side is the contribution of the electrons to the probe current. $T_{\mathrm{e}}$ is the electron temperature and $I_{\mathrm{sat}}^{0}$ the extrapolated value of the ion current at the floating potential $V_{\mathrm{fl}}$. It is taken as the Bohm value $^{3,4}$

$$
I_{\mathrm{sat}}^{0}=\frac{n}{2} c_{s} e A_{\mathrm{eff}}^{0},
$$

with $n$ the plasma density in the unperturbed plasma, $c_{s}$ $\approx \sqrt{T_{\mathrm{e}} / m_{i}}$ the ion sound speed and $A_{\text {eff }}^{0}$ the sheath surface at the floating potential.

Time average plasma parameters are obtained by fitting Eq. (A1) to the recorded current-voltage $(I-V)$ curves, averaged over several sweeping cycles. An example is shown in Fig. 11. The exponential growth of the electron current in Eq. (A1) is typically observed for $V_{\mathrm{pr}}$ up to $\approx V_{\mathrm{fl}}+2.5 \cdot T_{\mathrm{e}} / e$, such that a substantial fraction of the electron distribution contributes to the evaluation of $T_{\mathrm{e}}$. The plasma potential $V_{\mathrm{pl}}$ is determined from $V_{\mathrm{pl}}=V_{\mathrm{fl}}+\mu T_{\mathrm{e}} / e$, where $\mu$ can be deter-

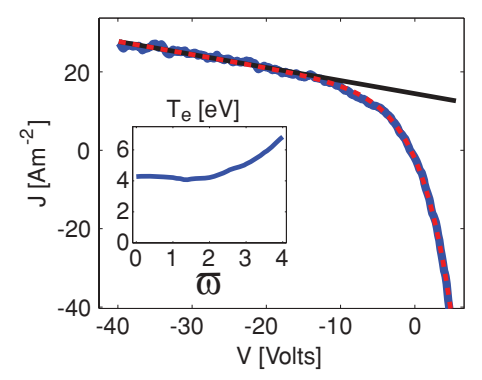

FIG. 11. (Color online) Example of a fit of Eq. (A1) to an $I-V$ curve. The blue solid line is the recorded $I-V$ characteristics, averaged over many sweeping cycles. The fit (red dashed line) is obtained here by first fitting the linear part of Eq. (A1) to the data (black solid line) and in a second step the exponential part. Inset: dependence of the evaluated electron temperature on $\varpi$, which is related to the upper voltage limit $V_{\max }$ used for the fit through $V_{\max }=V_{\mathrm{fl}}+\varpi \cdot T_{\mathrm{e}} / e$. mined experimentally for a given probe ${ }^{12}$ and is typically $\approx 3$ for hydrogen plasmas. ${ }^{3,4}$ Finally, the density $n$ is determined from Eq. (A2). For a cylindrical probe of radius $r_{p}$ that does not satisfy $r_{p} \gg \lambda_{D}$ and $r_{p} \gg \rho_{i}$ with $\lambda_{D}$ the Debye length and $\rho_{i}$ the ion Larmor radius, the estimate of $A_{\text {eff }}^{0}$ is subject to uncertainty. ${ }^{4}$ However, the evaluated density profile can be cross-checked with the experimentally determined location of the upper-hybrid resonance. ${ }^{12}$

Equations (A1) and (A2) (with $\alpha=0$ ) are derived from a 1D collisionless sheath model, assuming Maxwellian electrons, $T_{i}<T_{\mathrm{e}}$, electron and ion mean-free path $l_{e}, l_{i} \gg d$ with $d$ the diffusion length, ${ }^{4,5} r_{p} \gg \lambda_{D}$, and zero magnetic field. ${ }^{3,4}$ Further, a perfectly absorbing probe surface is assumed, i.e., no reflection or secondary emission of charged particles. Finally, the model requires that the fluctuations in the plasma (and the sweep frequency of the probe voltage) are slow enough such that the sheath is in equilibrium at all times. In the reminder of this section, we discuss the validity of Eqs. (A1) and (A2) for TORPEX plasmas.

The conditions $T_{i}<T_{\mathrm{e}}$ and $l_{e}, l_{i} \gg d$ are always satisfied in the electron heated TORPEX plasmas for typical neutral and plasma densities. The ion mean-free path $l_{i}$ for collisions with unlike species is limited by charge-exchange collisions with neutrals. With a momentum transfer cross section in the lab frame of $\approx 5 \times 10^{-19} \mathrm{~m}^{2}$ for $\mathrm{H}-\mathrm{H}^{+}$collisions ${ }^{25,26}$ and a typical neutral density of $5 \times 10^{18} \mathrm{~m}^{-3}$, we find $l_{i} \approx 0.4 \mathrm{~m}$. For electrons, the cross section for collisions with neutrals is $\approx 1 \times 10^{-19} \mathrm{~m}^{2}$ for $T_{\mathrm{e}}=5 \mathrm{eV}$ (Ref. 26) and we find $l_{e} \approx 2 \mathrm{~m}$. For a cylindrical probe, the diffusion length ${ }^{5}$ is given by $d \approx$ $r_{p} \ln \left(\pi l_{p} / 4 / r_{p}\right)$ with $l_{p}$ the probe length. $d$ measures thus a few times the probe radius and $l_{e}, l_{i} \gg d$ is well satisfied.

The extension of Eqs. (A1) and (A2) to cylindrical probes in magnetized plasmas with $\rho_{i}, \lambda_{D} \ll r_{p}$ can be justified by a quasi-collisionless model. ${ }^{4}$ The probe causes a depleted, quasi-neutral region along the magnetic field that needs to be refilled by an ionization source, cross-field diffusion or convection. If the length $L$ of this region along the field is shorter than the ion mean-free path $l_{i}$, Eqs. (A1) and (A2) are still valid when $A_{\mathrm{eff}}^{0}$ is taken as the projection of the probe surface along the magnetic field. An estimate of $L$ is given in Hutchinson's book, ${ }^{4} L \approx r_{p}^{2} c_{s} / D_{\perp}$, with $D_{\perp}$ the cross-field diffusion coefficient. Assuming Bohm diffusion, $D_{\text {Bohm }}=T_{e}[\mathrm{eV}] /(16 B)$, we find that $L<5 \mathrm{~cm}<l_{i}$ for all probe sizes used in TORPEX.

We typically have $\lambda_{D} \sim 0.1-0.2 \mathrm{~mm}$ and $\rho_{i} \lesssim 1.3 \mathrm{~mm}$ for $T_{i} \lesssim 1 \mathrm{eV}$ (hydrogen) in TORPEX. For a probe with $r_{p}=0.35 \mathrm{~mm}$ as used in the present work, we are thus not in the limit $\rho_{i}, \lambda_{D} \ll r_{p}$. Therefore, tests have been performed to compare measurements with probes of radius $r_{p}=0.3 \mathrm{~mm}$ and $r_{p}=3 \mathrm{~mm}$, respectively. In either case, the $I-V$ curves are well represented by Eq. (A1) and yield consistent values of $T_{\mathrm{e}}$ and $V_{\mathrm{ff}}$. The main difference is the reduction of the normalized slope $\alpha$ of the ion saturation current of approximately a factor of $1 / 2$ to $1 / 3$ for the larger probe. The deduction of the plasma density requires an estimate of $A_{\mathrm{eff}}^{0}$ which is subject to uncertainty (full or projection area). Further, we expect $A_{\text {eff }}^{0}$ to depend on $\lambda_{D}$ and thus on position. However, for a wide range of plasmas in TORPEX, $\lambda_{D}$ is fairely constant over the cross-section and one would expect to find similar 
density profiles with different probes, up to a constant factor of order unity. And this is in fact what is observed with the two different probe radii.

The assumption of a perfectly absorbing probe surface is violated when a significant fraction of impinging electrons is reflected or gives rise to secondary electron emission. For Maxwellian electrons, the effect of this is that the floating potential moves closer to the plasma potential, while the evaluation of $T_{\mathrm{e}}$ remains unaffected. ${ }^{1,3}$ The reflection of nonneutralized ions and ion-induced electron emission could also occur. This would alter the apparent ion current and add, besides the estimation of $A_{\text {eff }}^{0}$, additional uncertainty on the determination of density.

Departures from a Maxwellian electron distribution have been observed with an electrostatic energy-analyser in TORPEX. A suprathermal population representing a few percent of the total density was found around the upper-hybrid resonance. ${ }^{27}$ Such a suprathermal population can strongly effect the determination of the bulk electron temperature in plasmas where the $I-V$ curve can only be fitted up to the floating potential. ${ }^{28}$ In TORPEX, fitting the $I-V$ curves over voltage ranges with upper bounds ranging from $V_{\mathrm{fl}}+0.5 \cdot T_{\mathrm{e}}^{\circ} / e$ until $V_{\mathrm{fl}}+2.5 \cdot T_{\mathrm{e}}^{\circ} / e$, where $T_{\mathrm{e}}^{\circ}$ is a first estimate of $T_{\mathrm{e}}$, results in similar electron temperatures. This is shown in the example in Fig. 11, with a fit to the recorded $I-V$ curve. The inset shows the temperature obtained from performing the fit in a voltage range between $-40 \mathrm{~V}$ and $V_{\mathrm{fl}}+\varpi \cdot T_{\mathrm{e}}^{\circ} / e$, with similar results for different values of the parameter $\varpi$ as long as $0 \leq \varpi \lesssim 2.5$. This gives us confidence that our evaluation of the electron temperature is not strongly affected by a weak suprathermal population.

The application of a dc sheath theory requires that fluctuations are slow enough such that the ions have time to respond. The frequency limit for this is usually assumed to be of the order of the ion plasma frequency. ${ }^{2}$ In TORPEX, the ion plasma frequency lies in the range $\approx 10-20 \mathrm{MHz}$, which is well above observed fluctuation spectra.

Modifications to Eqs. (A1) and (A2) can still arise due to sheath capacitance or ion-polarization currents. ${ }^{6}$ However, these effects should not contribute to the time averaged $I-V$ curve.

The presence of parallel and cross-field flows can also give corrections to the ion current. ${ }^{29-31}$ We have typically parallel and cross-field flows $\lesssim 40 \%$ and $\lesssim 5 \%$ of $c_{s}$ over most of the cross section in hydrogen plasmas. ${ }^{32}$ From the cited references, we expect small corrections to the ion current for such values of the flows. We note, however, that these works do not quite apply to our parameter regime.

A problem that can arise due to the averaging of $I-V$ curves is that a time averaged $I-V$ curve is not identical to the $I-V$ curve of the time averaged plasma parameters. This adds additional uncertainty to this kind of measurements. ${ }^{2,33}$ The good agreement between time averaged profiles obtained in this work with swept probes and with the triple probe method indicate however that this is not an important issue here.

Finally, another practical difficulty is that of impurity layers on the probe surface. It is well known that dirty probe surfaces can distort the $I-V$ curve and give false plasma parameters. ${ }^{1,34} \mathrm{~A}$ sign of dirty probes can be a hysteresis, i.e., a different $I-V$ curve for the upwards and downwards sweep phase. ${ }^{35,36}$ Indeed, hysteresis can be observed on newly installed probes on TORPEX. Keeping the probes in ion saturation current during plasma discharges of several minutes sputter cleans the probe and removes this hysteresis permanently. In certain plasmas, impurity layers can reform quickly. Therefore, tests have been performed in TORPEX where, instead of the usual continuous sawtooth sweep, the probe was held on a strong negative potential $(-40$ or $-80 \mathrm{~V})$ during $20 \mathrm{~ms}$ prior to each sweep cycle. This has not shown any significant effect on the $I-V$ curve.

${ }^{1}$ F. F. Chen, Plasma Diagnostic Techniques, edited by R.H. Huddlestone and S. L. Leonard (Academic, New York, 1965).

${ }^{2}$ J. D. Swift and M. J. R. Schwar, Electrical Probes for Plasma Diagnostics (Lliffe, London, 1970)

${ }^{3}$ P. C. Stangeby, The Plasma Boundary of Magnetic Fusion Devices (Institute of Physics, London, 2000).

${ }^{4}$ I. H. Hutchinson, Principles of Plasma Diagnostics, 2nd ed. (Cambridge University Press, Cambridge, England, 2002).

${ }^{5}$ V. I. Demidov, S. Ratynskaia, and K. Rypdal, Rev. Sci. Instrum. 73, 3409 (2002).

${ }^{6}$ G. Chiodini, C. Riccardi, and M. Fontanesi, Rev. Sci. Instrum. 70, 2681 (1999).

${ }^{7}$ R. B. Lobbia and A. D. Gallimore, Rev. Sci. Instrum. 81, 073503 (2010).

${ }^{8}$ S. Chen and T. Sekiguchi, J. Appl. Phys. 36, 2363 (1965).

${ }^{9}$ H. Y. W. Tsui, R. D. Bengtson, G. X. Li, H. Lin, M. Meier, C. P. Ritz, and A. J. Wootton, Rev. Sci. Instrum. 63, 4608 (1992).

${ }^{10}$ A. Fasoli, B. Labit, M. McGrath, S. H. Müller, G. Plyushchev, M. Podestà, and F. M. Poli, Phys. Plasmas 13, 055902 (2006); A. Fasoli, A. Burckel, L. Federspiel, I. Furno, K. Gustafson, D. Iraji, B. Labit, J. Loizu, G. Plyushchev, P. Ricci, C. Theiler, A. Diallo, S. H. Müller, M. Podestà, and F. Poli, Plasma Phys. Contr. F. 52, 124020 (2010).

${ }^{11}$ E. O. Johnson and L. Malter, Phys. Rev. 80, 58 (1950).

${ }^{12}$ M. Podestà, A. Fasoli, B. Labit, M. McGrath, S. H. Müller, and F. M. Poli, Plasma Phys. Contr. F. 47, 1989 (2005).

${ }^{13}$ J. M. Beall, Y. C. Kim, and E. J. Powers, J. Appl. Phys. 53, 3933 (1982).

${ }^{14}$ F. M. Poli, P. Ricci, A. Fasoli, and M. Podestà, Phys. Plasmas 15, 032104 (2008).

${ }^{15}$ P. Ricci and B. N. Rogers, Phys. Rev. Lett. 104, 145001 (2010).

${ }^{16}$ I. Furno, B. Labit, M. Podestà, A. Fasoli, S. H. Müller, F. M. Poli, P. Ricci, C. Theiler, S. Brunner, A. Diallo, and J. Graves, Phys. Rev. Lett. 100, 055004 (2008).

${ }^{17}$ C. Theiler, A. Diallo, A. Fasoli, I. Furno, B. Labit, M. Podestà, F. M. Poli, and P. Ricci, Phys. Plasmas 15, 042303 (2008).

${ }^{18}$ I. Furno, B. Labit, A. Fasoli, F. M. Poli, P. Ricci, C. Theiler, S. Brunner, A. Diallo, J. P. Graves, M. Podestà, and S. H. Müller, Phys. Plasmas 15, 055903 (2008).

${ }^{19}$ E. J. Powers, Nucl. Fusion 14, 749 (1974).

${ }^{20}$ S. H. Müller, A. Diallo, A. Fasoli, I. Furno, B. Labit, and M. Podestà, Phys. Plasmas 14, 110704 (2007).

${ }^{21}$ M. Podestà, A. Fasoli, B. Labit, I. Furno, P. Ricci, F. M. Poli, A. Diallo, S. H. Müller, and C. Theiler, Phys. Rev. Lett. 101, 045001 (2008).

${ }^{22}$ P. Ricci, C. Theiler, A. Fasoli, I. Furno, B. Labit, S. H. Müller, M. Podestà, and F. M. Poli, Phys. Plasmas 16, 055703 (2009).

${ }^{23}$ P. Ricci, C. Theiler, A. Fasoli, I. Furno, K. Gustafson, D. Iraji, and J. Loizu, "Methodology for turbulence code validation: quantification of simulationexperiment agreement and application to the torpex experiment," Phys. Plasmas (submitted)

${ }^{24}$ C. Theiler, I. Furno, P. Ricci, A. Fasoli, B. Labit, S. H. Müller, and G. Plyushchev, Phys. Rev. Lett. 103, 065001 (2009).

${ }^{25}$ A. E. Glassgold, P. S. Krstić, and D. R. Schultz, Astrophys. J. 621, 808 (2005).

${ }^{26}$ Atomic and Molecular Processes in Fusion Edge Plasmas, edited by R. K. Janev (Plenum, New York, 1995).

${ }^{27}$ M. Podestà, A. Diallo, A. Fasoli, I. Furno, B. Labit, S. H. Müller, and F. M. Poli, Plasma Phys. Contr. F. 49, 175 (2007).

${ }^{28}$ P. C. Stangeby, Plasma Phys. Contr. F. 37, 1031 (1995).

${ }^{29}$ P. C. Stangeby and J. E. Allen, J. Plasma Phys. 6, 19 (1971).

${ }^{30}$ J. C. McMahon, G. Z. Xu, and J. G. Laframboise, Phys. Plasmas 12, 062109 (2005). 
${ }^{31}$ I. H. Hutchinson, Phys. Rev. Lett. 101, 035004 (2008).

${ }^{32}$ B. Labit, C. Theiler, A. Fasoli, I. Furno, and P. Ricci, "Blob-induced toroidal flows in simple magnetized plasmas," Phys. Plasmas (submitted).

${ }^{33}$ D. L. Rudakov, J. A. Boedo, R. A. Moyer, P. C. Stangeby, A. McLean, and J. G. Watkins, Rev. Sci. Instrum. 75, 4334 (2004).
${ }^{34}$ J. F. Waymouth, J. Appl. Phys. 30, 1404 (1959).

${ }^{35}$ K. Oyama, Planet. Space Sci. 24, 183 (1976).

${ }^{36}$ W. E. Amatucci, P. W. Schuck, D. N. Walker, P. M. Kintner, S. Powell, B. Holback, and D. Leonhardt, Rev. Sci. Instrum. 72, 2052 (2001). 